\title{
Diagnosis of mitral valve aneurysm by transoesophageal echocardiography
}

David B Northridge, James P Gnanapragasam, Alan B Houston

\begin{abstract}
In a patient with mitral valve aneurysm precordial echocardiography suggested a mistaken diagnosis of infective endocarditis. Transoesophageal echocardiographic examination established the correct diagnosis, which was subsequently confirmed at operation. Transoesophageal echocardiography gives better resolution of lesions associated with the mitral valve than precordial examination and may improve the diagnostic accuracy.
\end{abstract}

True aneurysms, or diverticulae, of the mitral valve are a rare cause of mitral regurgitation. They are most common in patients with infective endocarditis affecting the aortic valve, ${ }^{1-7}$ but they may also present with chronic mitral regurgitation, ${ }^{8}$ or as a complication of mitral valve prolapse. ${ }^{9}$ The precordial echocardiographic features of mitral valve aneurysms are non-specific, ${ }^{5810}$ and can be misinterpreted as a large vegetation, cystic left atrial myxoma, or degeneration in association with a prolapsing leaflet. Delay in making the correct diagnosis increases the risk of infection, embolisation, or rupture of the aneurysm; these complications can be prevented by surgery to repair or replace the valve. ${ }^{5}$ We report a patient in whom a mitral valve aneurysm was diagnosed by transoesophageal echocardiography.

\section{Case report}

A 43 year old woman was admitted to hospital with a purpuric rash. On direct questioning she also gave a six month history of night sweats. She did not report any previous cardiac history. Multiple sclerosis had been diagnosed three years earlier after two separate presentations with neurological symptoms, both of which resolved completely with steroid treatment. She was not on any long term drug treatment. No cardiac murmurs had been noticed on previous hospital visits.

We found several purpuric skin lesions on her trunk and upper limbs. There were no other peripheral signs of infective endocarditis and her temperature, pulse, and blood pressure were normal. A loud pansystolic murmur (grade 3) was present at the apex.

The haemoglobin concentration, white cell count, and platelet count were normal as was a full coagulation screen. Six blood cultures were negative, as were serological tests for a wide range of viruses. Chest radiography and electrocardiography were normal. Precordial echocardiography showed a mass attached to the posterior leaflet of the mitral valve that was thought to be a large vegetation. Colour flow Doppler examination showed moderately severe mitral regurgitation. Infective endocarditis was diagnosed and treatment with an intravenous antibiotic was started. She was subsequently referred to the Western Infirmary for further cardiological assessment.

In systole a transoesophageal echocardiographic examination showed a large cystic lesion in the left atrium attached to the posterior leaflet of the mitral valve (fig 1). In diastole the cyst collapsed and became much less prominent (fig 2). Colour flow mapping

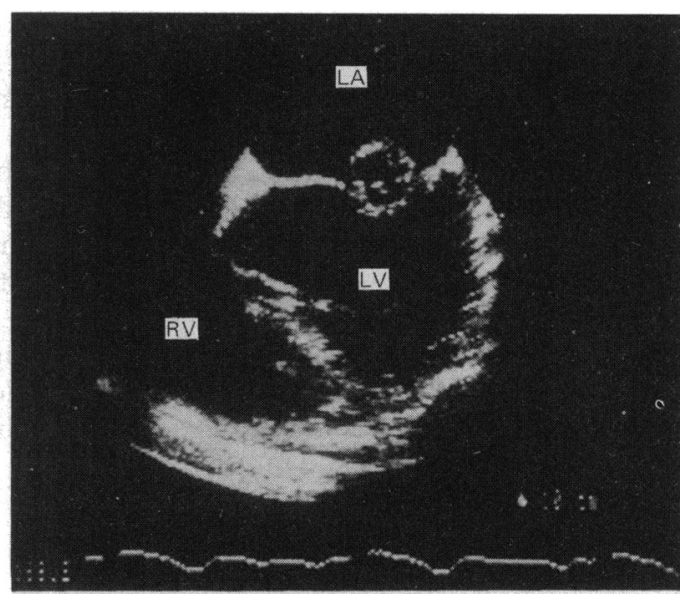

Figure 1 Transoesophageal echocardiogram in systole showing a large aneurysm on the mitral valve. $L A$, left atrium; $L V$, left ventricle; $R V$, right ventricle

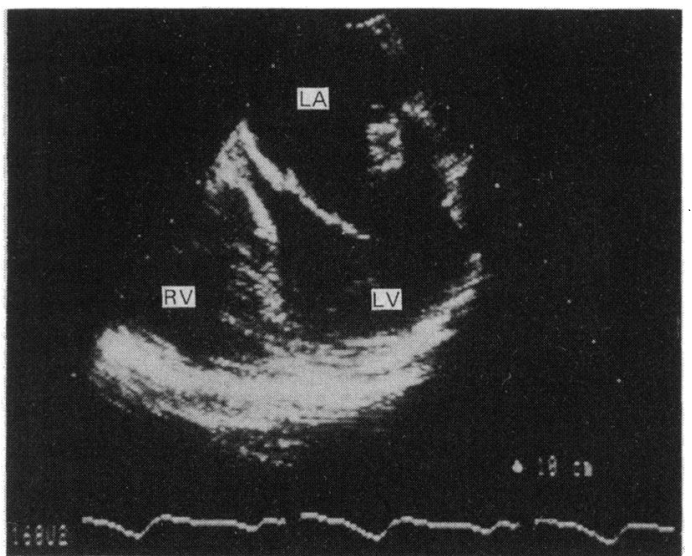

Figure 2 Transoesophageal echocardiogram in diastole showing collapse of the aneurysm and its origin on the posterior leaflet. L $A$, left atrium; $L V$, left ventricle; $R V$, right ventricle 
showed an eccentric jet of mitral regurgitation directed towards the interatrial septum. Cardiac catheterisation with left ventriculography subsequently confirmed that this lesion was a mitral valve aneurysm that communicated with the left ventricular cavity. The cyst was excised on cardiopulmonary bypass, and the posterior leaflet of the mitral valve was repaired without the need for valve replacement.

\section{Discussion}

Aneurysm of the mitral valve is more commonly reported as a complication of infective endocarditis affecting primarily the aortic valve. ${ }^{1-7}$ In other reports, ${ }^{8}$ as in our patient, there was no previous history of endocarditis, though a subclinical infection cannot be excluded. Aneurysms of the mitral valve seem to be more common on the anterior leaflet than the posterior mitral. ${ }^{12}$

Diagnosis of mitral valve aneurysm by precordial cross sectional echocardiography has been reported before..$^{23-9}$ Our patient, however, shows that precordial echocardiography may not be diagnostic in this condition and can be misleading especially when the posterior leaflet is affected. ${ }^{8-10}$ Mitral valve aneurysm may be confused with cystic degeneration of the valve, a cystic atrial myxoma, or a large vegetation. ${ }^{10}$ Transoesophageal echocardiography can distinguish between these conditions. Because the oesophagus lies immediately behind the left atrium it provides an excellent echocardiographic window and allows a high ultrasound frequency to be used. This gives much better resolution of abnormalities in the region of the mitral valve than precordial echocardiography. ${ }^{11}$ We are aware of only one previous report of transoesophageal echocardiographic diagnosis of a mitral valve aneurysm, where the findings resembled those in our patient. ${ }^{10}$

Aneurysm of the mitral valve should be considered in the differential diagnosis of mass lesions seen on the atrial side of the mitral valve at precordial echocardiography. A transoesophageal examination may be needed in such patients. When the characteristic features described above are present a diagnosis of mitral valve aneurysm can be made without recourse to cardiac catheterisation.

We thank Dr John Reid and Mr Alan Faichney for permission to report this case.

1 Saphir O, Leroy EP. True aneurysms of the mitral valve in ubacute bacterial endocarditis. Am J Pathol 1948;24: 83-95.

2 Enia F, Celona G, Filippone V. Echocardiographic detection of mitral valve aneurysm in patient with infective endocardits. Br Heart J 1983;49:98-100.

3 Decroly P, Vandenbossche JL, Englert M. Anterior mitral valve aneurysm perforation secondary to aortic valve endocarditis detected by Doppler colour flow mapping. Eur Heart J 1989;10:186-9.

4 English TAH, Honey M, Cleland WP. Ruptured true aneurysm of mitral valve. A complication of aortic valve endocarditis. Br Heart $J$ 1972;34:434-6.

5 Reid CL, Chandraratna AN, Harrison E, et al. Mitral valve aneurysm: clinical features, echocardiographic-pathologic aneurysm: clinical features, echocardiographic-

6 Vandenbossche JL, Hartenberg D, Leclerc JL. Mitral valve aneurysm formation documented by cross-sectional echocardiography. Eur Heart $J$ 1986;7:171-5.

7 De Luca I, Colonna L. Echocardiographic diagnosis of mitral valve aneurysm. Eur J Cardiol 1980;11:325-30.

8 Lewis BS, Colsen PR, Rosenfield T, McKibben JK, Barlow JB. An unusual case of mitral valve aneurysm: two dimensional echocardiographic and cineangiocardiographic features. Am J Cardiol 1982;49:1293-6.

9 Ruckel A, Erbel R, Henkel B, Kramer B, Meyer J. Mitral valve aneurysm revealed by cross-sectional echocardiography in a patient with mitral valve prolapse. Int $\mathrm{J}$ Cardiol 1984;6:633-7.

10 Teskey RJ, Chan KL, Beanlands DS. Diverticulum of the mitral valve complicating bacterial endocarditis: diagnosis mitral valve complicating bacterial endocarditis: diagnosis by transesophage

11 Roelandt JRTC, Sutherland GR. Oesophageal echocardiography. Br Heart $J$ 1988;60:1-3. 